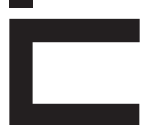

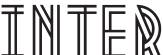

$\mathbb{E}(\mathbb{E}(\mathbb{E}$

CIENTÍFICAS

EXATAS E TECNOLÓGICAS

ISSN IMPRESSO - 2359-4934

E-ISSN - 2359-4942

DOI 10.17564/2359-4934.2016v2n1p41-50

\title{
O EMPREGO DE SOLUÇ̃̃ES EXATAS PARA A EQUAÇÃO DE LAPLACE NO PLANO PARA O PROJETO DE SUPERFÍCIES ALETADAS
}

Vinicius G. Ribeiro

Volnei Borges ${ }^{3}$

\section{RESUMO}

0 presente trabalho apresenta um método simples para projeto de superfícies estendidas - também conhecidas como superfícies aletadas. Este esquema é baseado em soluções analíticas para a equação Laplace bidimensional com uma condição de contorno de primeiro tipo, que é obtido por ajuste de curva. Os dados experimentais consistem em medições de temperatura ao longo da superfície nua.
Jorge R. Zabadal ${ }^{2}$

\section{PALAVRAS-CHAVE}

Projeto de superfícies aletadas. Equação de condução do calor. Regime estacionário. Soluções exatas. Desempenho computacional. 


\section{ABSTRACT}

This work presents a straightforward method to perform the design of extended surfaces. This scheme is based on analytical solutions to the two dimensional Laplace equation with a first kind boundary condition which is obtained by curve fitting. The experimental data consists in temperature measurements along the bared surface.

\section{RESUMEN}

Este artículo presenta un método simple para proyecto de superficies extendidas. Este esquema se basa en soluciones analíticas para la ecuación de Laplace bidimensional con una condición de contorno de primer tipo, que se obtiene por ajuste de curvas. Los datos experimentales consisten en mediciones de temperatura a lo largo de la superficie desnuda.

\section{KEYWORDS}

Finned surfaces project. Heat conduction equation. Steady state. Exact solutions. Computational performance.

\section{PALABRAS CLAVE}

Proyecto de superficies con aletas; Ecuación de conducción de calor; Estado estacionario; Soluciones exactas; Rendimiento computacional. 


\section{INTRODUÇ̄̃̃O}

Em qualquer aparelho cujo funcionamento seja prejudicado pelo superaquecimento, a inserção ou soldagem de várias placas metálicas visando a dissipação do calor para o ar estagnado constitui uma solução pratica de baixo custo, para evitar danos oriundos do aumento excessivo de temperatura. 0 projeto de superfícies aletadas concebidas com o fim de dissipar calor no ar atmosférico envolve uma série de cálculos que tem por objetivo estabelecer os valores locais da temperatura em cada ponto dessas placas.

Esses valores locais são empregados para estimar a quantidade de calor fornecido por uma fonte primária para a superfície aletada (como exemplo de fontes primarias, temos CPU, motores de geladeiras ou motor de motocicleta).

Ocorre que o cálculo da quantidade de calor transferida da fonte primária para as aletas e das aletas par ao ar - grandeza denominada carga térmica do sistema - frequentemente se torna inviável devido ao número excessivo de parcelas que constitui a solução analítica do problema. Isso ocorre porque essas soluções analíticas são expressas em séries de Fourier, que demandam extremamente elevado número de componentes para produzir um perfil suave de temperatura.

Embora exista uma outra abordagem para a solução desse problema, baseada em métodos numéricos - diferenças finitas, elementos finitos, elementos de contorno etc. (GREENSPAN; CASULI, 1988; CARNAHAM, 1990; MALISKA, 1995) -, essas formulações demandam tempo de processamento relativamente elevado, de modo que se faz necessário elaborar método eficiente para viabilizar o projeto de várias unidades de dissipação de calor em curto espaço de tempo.

Além disso, o dimensionamento de superfícies estendidas é usualmente efetuado a partir de soluções exatas para problemas unidimensionais em transfe- rência de calor. Essas soluções levam em consideração apenas a propagação do calor no sentido longitudinal das aletas soldadas sobre superfícies cuja dissipação é ineficiente (HOLMAN, 2009). Entretanto, os maiores gradientes de temperatura ocorrem no sentido transversal, isto é, da interface de soldagem para a borda principal da aleta.

No trabalho proposto, o dimensionamento de aletas é efetuado a partir de soluções exatas da equação de Laplace no plano, que fornece soluções bidimensionais em regime estacionário para o problema de transferência de calor por condução (POLYANIN; ZAITSEV, 2004). Esse método foi elaborado e otimizado após analisar não apenas estratégias para dimensionamento de aletas, mas também trocadores duplo-tubo e casco-tubo (BARTLETT, 1996; RAFFERTY; CULVER, 1998; INDUSTRIAL HEAT TRANSFER, 2011; MUKHERJEE, 1998).

0 presente trabalho está estruturado da seguinte forma: a seção dois apresenta o modelo matemático utilizado; na seção três é empregada a equação de Poison - válida quando há fontes térmicas dentro do domínio estudado. Resultados obtidos e conclusão são apresentados na seção quatro.

\section{MODELO MATEMÁTICO UTILIZADO}

Considere-se inicialmente a equação de Laplace no plano, dada por

$$
\frac{\partial^{2} f}{\partial y^{2}}+\frac{\partial^{2} f}{\partial x^{2}}=0
$$

Essa equação pode ser reescrita em termos de variáveis complexas, mudança esta feita pela introdução da variável $z$ e de seu conjugado $\bar{z}$ : 


$$
\begin{aligned}
& z=x+i y \\
& \bar{z}=x-i y \cdot(2)
\end{aligned}
$$

Aplicando a regra da cadeia a fim de redefinir as derivadas espaciais em função das novas variáveis, resulta

$$
\begin{aligned}
& \frac{\partial^{2} f}{\partial x^{2}}=\frac{\partial g}{\partial z}+\frac{\partial g}{\partial \bar{z}}=2 \frac{\partial^{2} f}{\partial z \partial \bar{z}}+\frac{\partial^{2} f}{\partial z^{2}}+\frac{\partial^{2} f}{\partial \bar{z}^{2}} \\
& \frac{\partial^{2} f}{\partial x^{2}}=\frac{\partial g}{\partial z}+\frac{\partial g}{\partial \bar{z}}=2 \frac{\partial^{2} f}{\partial z \partial \bar{z}}+\frac{\partial^{2} f}{\partial z^{2}}+\frac{\partial^{2} f}{\partial \bar{z}^{2}} \\
& \frac{\partial f}{\partial y}=\frac{\partial f}{\partial z} \frac{\partial z}{\partial y}+\frac{\partial f}{\partial \bar{z}} \frac{\partial \bar{z}}{\partial y}=i \frac{\partial f}{\partial z}-i \frac{\partial f}{\partial \bar{z}} \\
& \frac{\partial^{2} f}{\partial y^{2}}=2 \frac{\partial^{2} f}{\partial z \partial \bar{z}}-\frac{\partial^{2} f}{\partial z^{2}}-\frac{\partial^{2} f}{\partial^{2}}
\end{aligned}
$$

Substituindo as expressões (4) e (6) na equação de Laplace, obtém-se

$$
\nabla^{2} f=\frac{\partial^{2} f}{\partial x^{2}}+\frac{\partial^{2} f}{\partial y^{2}}=4 \frac{\partial^{2} f}{\partial z \partial \bar{z}}=0
$$

ou

$$
\frac{\partial^{2} f}{\partial z \partial \bar{z}}=0
$$

A solução geral dessa equação é obtida de imediato. Ao decompor (8) no sistema

$$
\frac{\partial f}{\partial z}=q
$$

$$
\frac{\partial q}{\partial \bar{z}}=0
$$

De acordo com (10), q não depende do conjugado de $z$, e portanto $q=c(z)$, sendo $c$ uma função arbitrária de seu argumento. Assim, a equação (9) se reduz a

$$
\frac{\partial f}{\partial z}=c(z)
$$

Naturalmente, a integração de (11) fornece uma nova função arbitrária do mesmo argumento, de modo que

$$
f=\int c(z) d z+b(\bar{z})=a(z)+b(\bar{z})
$$

Nessa equação, b representa outra função arbitrária, que pertence ao espaço nulo do operador derivada em relação a z. Retornando às variáveis independentes originais, isto é, considerando a definição das variáveis complexas em termos das coordenadas cartesianas, obtém-se

$$
f=a(x+i y)+b(x-i y)
$$

Uma vez que no problema proposto existe apenas uma condição de contorno relevante a considerar, é necessário especificar apenas uma das funções arbitrárias que compõem a solução geral. Desse modo, a solução particular

$$
f=a(x+i y)(14)
$$

possui graus de liberdade suficientes para satisfazer à equação alvo e à condição de contorno que especifica o perfil unidimensional de temperaturas na base da aleta, isto é, $\mathrm{T}(\mathrm{x}, 0)$. 
É importante observar que a equação (14) informa apenas que qualquer função do argumento $x+$ iy constitui uma solução exata da equação de Laplace no plano. Assim, o processo de obtenção da distribuição de temperaturas bidimensional em regime estacionário pode ser reduzido a um roteiro composto de cinco passos:

i - medir a temperatura em alguns pontos ao longo da linha sobre a qual se deseja soldar a aleta sobre a superfície de interesse;

ii - construir uma tabela contendo as coordenadas $x$ e suas respectivas temperaturas medidas;

iii - ajustar uma função a partir da tabela de pontos, obtendo o perfil unidimensional de temperaturas $f(x)$ na base da aleta;

iv - efetuar a mudança de variáveis $\mathrm{x} \rightarrow \mathrm{x}+\mathrm{iy}$;

v - extrair a parte real da função obtida.

A execução da última etapa se faz necessária porque a temperatura é uma variável real.

Em resumo, a distribuição de temperaturas que constitui a solução exata para o problema de dimensionamento de superfícies aletadas é definida como

$$
T=\operatorname{Re}[f(x+i y)],(15)
$$

onde $f$ representa a função que descreve o perfil de temperaturas na base da aleta, isto é, que satisfaz à condição de contorno na interface $y=0$, dada por $T(x, 0)=f(x)$.

\section{MODELO ADICIONAL: A EQUAÇ̃̃O DE POISON}

A equação de Poisson consiste na versão não-homogênea da equação de Laplace, sendo portanto definida como

$$
\frac{\partial^{2} f}{\partial y^{2}}+\frac{\partial^{2} f}{\partial x^{2}}=Q
$$

é válida quando existem fontes térmicas no interior do domínio. Nesta equação, Q representa uma fonte interna de energia. Uma vez mapeado o operador Laplaciano na forma complexa, é preciso reescrever a função fonte original $\mathrm{Q}(\mathrm{x}, \mathrm{y})$ como um novo termo não-homogêneo. Em outras palavras, é necessário expressar as coordenadas $x$ e y em função das variáveis complexas ze $z$.

Para que a mudança de variável seja feita basta isolar as variáveis do sistema (3), obtendo assim as relações de x e y em função de $z$ e $z$. Desta forma, podemos expressar $x$ e y como

$$
\begin{aligned}
& x=\frac{z+\bar{z}}{2} \\
& y=\frac{i(\bar{z}-z)}{2}
\end{aligned}
$$

Agora, então, pode-se expressar a equação de Poisson em termos das variáveis complexas da seguinte forma

$$
\nabla^{2} f=4 \frac{\partial^{2} f}{\partial z \partial \bar{z}}=q(z, \bar{z})
$$

A partir da equação (18), pode-se obter uma solução imediata, simplesmente pela dupla integração do termo fonte $q(z, \bar{z})$, somada ao espaço nulo do operador:

$$
\mathrm{f}=\frac{1}{4} \iint \mathrm{q}(\mathrm{z}, \overline{\mathrm{z}}) \mathrm{dz} d \overline{\mathrm{z}}+\mathrm{f}_{1}(\overline{\mathrm{z}})+\mathrm{f}_{2}(\mathrm{z})
$$

onde os termos $f_{1}(\bar{z})+f_{2}(z)$ correspondem ao espaço nulo do operador Laplaciano, já que 


$$
\nabla^{2}\left(f_{1}(\bar{z})+f_{2}(z)\right)=4 \frac{\partial^{2} f}{\partial z \partial \bar{z}}\left(f_{1}(\bar{z})+f_{2}(z)\right)=0
$$

\section{RESULTADOS OBTIDOS E CONCLUSÃO}

Solução analítica obtida na Seção 2 é utilizada a seguir para resolver um problema de contorno relativo à transferência de calor por condução em superfícies estendidas. A Tabela 1 mostra os valores das temperaturas ao longo de uma linha reta traçada sobre a superfície de um duto cilíndrico no interior do qual circula um gás, cuja temperatura de entrada é de aproximadamente $100^{\circ} \mathrm{C}$. A Tabela mostra valores de temperatura tomados sobre a superfície externa da tubulação, para uma temperatura ambiente em torno de $25^{\circ} \mathrm{C}$.

\begin{tabular}{|c|c|}
\hline \multicolumn{2}{|c|}{ Tabela 1 - Posição $\times$ Temper } \\
\hline $\mathbf{x ( c m )}$ & $\mathbf{T}\left({ }^{\circ} \mathbf{C}\right)$ \\
\hline 0 & 98.9 \\
\hline 1 & 82.1 \\
\hline 2 & 69.2 \\
\hline 3 & 59.2 \\
\hline 4 & 51.4 \\
\hline 5 & 45.4 \\
\hline
\end{tabular}

Fonte: Dados da pesquisa.

A Tabela 1 foi utilizada para obter uma função de ajuste que representa o perfil unidimensional de temperatura ao longo da superfície externa. A função obtida, dada por

$$
f=25.2317+e^{4.3028-0.2572 x}
$$

Apresenta desvio quadrático médio inferior a 1\% em relação aos dados tabelados. Efetuando a substituição $\mathrm{x} x+$ iy sobre a função ajustada e extraindo a parte real da função resultante, encontra-se

$$
f=25.2317+e^{4.3028-0.2572 x} \cos (0.2572 y)
$$

Obtém-se, assim, a distribuição de temperaturas bidimensional em regime estacionário, para qualquer ponto de uma aleta cuja base está sujeita à condição de contorno definida como

$$
T(x, 0)=25.2317+e^{4.3028-0.2572 x}
$$

A Figura 1 mostra o mapa de temperaturas sobre toda a extensão da aleta, enquanto a Figura 2 apresenta a respectiva superfície $\mathrm{T}(\mathrm{x}, \mathrm{y})$ em perspectiva.

Figura 1 - Distribuição de temperaturas obtida a partir da condição de contorno definida pela equação (23)

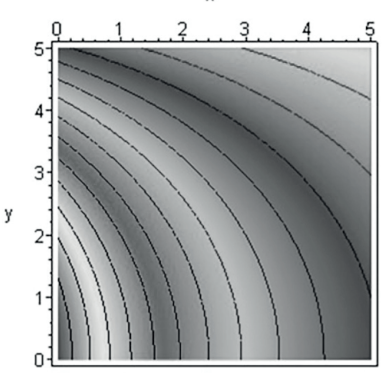

Fonte: Dados da pesquisa.

Figura 2 - Distribuição de temperaturas em perspectiva

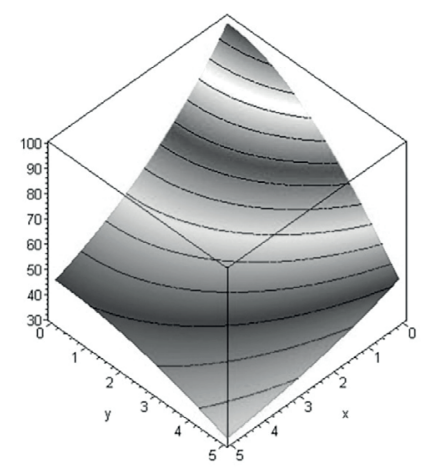

Fonte: Dados da pesquisa. 
A partir da solução obtida, o dimensionamento da superfície estendida é efetuado como segue.

I - Delimitação da área útil da aleta para fins de dissipação térmica. Consiste em traçar uma reta vertical e uma horizontal que delimitem um retângulo para o qual a diferença entre qualquer temperatura local e a temperatura ambiente seja significativa. No trabalho proposto, o limite inferior adotado para a essa diferença entre temperaturas é de aproximadamente $30^{\circ} \mathrm{C}$, conforme apresentado na Figura 3.

Figura 3 - Aleta delimitada segundo o critério definido no passo I

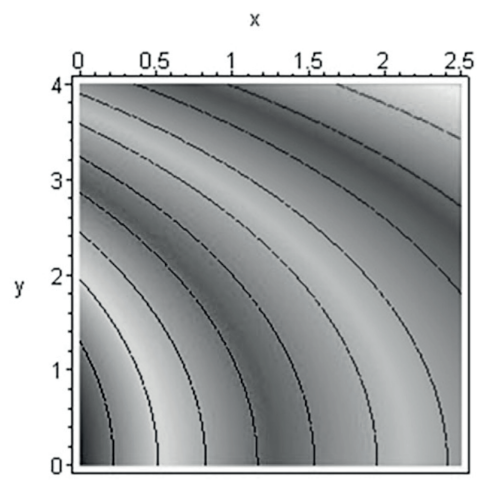

Fonte: Dados da pesquisa.

II - Utilizando a quantidade de energia a ser retirada para que o gás saia do duto à temperatura desejada, obtém-se a área mínima de troca térmica, por meio da relação

$$
m c_{p}\left(T_{e}-T_{s}\right)=h \int_{0}^{a} \int_{0}^{b}\left(T-T_{a m b}\right) d x d y
$$

Nessa equação, a e b são as dimensões da placa e o coeficiente de película corresponde ao do ar estagnado. As propriedades físicas e parâmetros de entrada para o problema são apresentados na Tabela 2.
Tabela 2 - Dados de entrada do problema

\begin{tabular}{|c|c|c|c|c|}
\hline $\mathbf{m}(\mathbf{k g} / \mathbf{s})$ & $\mathbf{C}_{\mathbf{p}}\left(\mathbf{k J} / \mathbf{k g}^{\mathbf{0}} \mathbf{C}\right)$ & $\mathbf{T}_{\mathbf{e}}\left({ }^{\circ} \mathbf{C}\right)$ & $\mathbf{T}_{\text {amb }}\left({ }^{\circ} \mathbf{C}\right)$ & $\begin{array}{c}\mathbf{H}(\mathbf{W} / \\
\left.\mathbf{c m}^{\mathbf{2}} \mathbf{C}\right)\end{array}$ \\
\hline 0.001 & 1000 & 100 & 25 & 0.05 \\
\hline
\end{tabular}

Fonte: Dados da pesquisa

III - Empregando o mesmo coeficiente de película utilizado na etapa anterior, conforme a Tabela 2, é estimada a quantidade de energia térmica dissipada pela aleta já limitada por meio do procedimento descrito no passo I. A quantidade de calor dissipada é obtida por meio de integração direta.

IV - Cálculo do número mínimo de aletas necessário para totalizar a área de dissipação exigida pelo critério estabelecido no passo II.

No caso específico do problema apresentado, a área total de troca em função da temperatura de saída desejada é apresentada na Tabela 3.

Tabela 3 - temperatura de saída do gás $\mathrm{x}$ área mínima requerida para dissipação térmica

\begin{tabular}{|c|c|}
\hline $\mathbf{T}\left({ }^{\circ} \mathbf{C}\right)$ & $\mathbf{A}\left(\mathbf{c m}^{2}\right)$ \\
\hline 25 & 16.4 \\
\hline 30 & 15.3 \\
\hline 35 & 14.2 \\
\hline 40 & 13.2 \\
\hline 45 & 12.1 \\
\hline 50 & 11.0 \\
\hline 55 & 9.9 \\
\hline
\end{tabular}

Fonte: Dados da pesquisa

Nesse ponto, o leitor familiarizado com projeto de equipamentos poderia argumentar que a condição de contorno na base da aleta muda a cada nova placa soldada sobre a superfície. Assim, o processo de dimensionamento deveria, a princípio, ser efetuado por meio de um método iterativo. 
Entretanto, é importante observar que a condição de contorno mais pessimista possível é precisamente aquela correspondente ao perfil de temperaturas na superfície sem aletas. Em outras palavras, parece razoável supor que a superfície aletada resultaria superdimensionada caso um processo iterativo não fosse empregado a partir do roteiro proposto. Entretanto, ocorre que uma estratégia bastante eficiente para evitar o superdimensionamento das aletas consiste justamente em estabelecer limites de corte como o proposto no Passo I. Esse critério de mínima diferença local entre as temperaturas ambiente e da placa torna o processo de dimensionamento bastante simples, uma vez que o esforço computacional requerido para a obtenção dos mapas de temperatura é extremamente reduzido.

O tempo de processamento requerido para a obtenção da solução e dos respectivos mapas de temperaturas foi de aproximadamente 2 segundos, em equipamento de baixo custo (AMD - Sempron 3100, com 512Mb de RAM). 0 modelo de Ozisik (1993) tem tempo de processamento de 20.000 segundos, no mesmo equipamento - o qual emprega aproximadamente 1500 termos para cada somatório, o que onera o processamento. Ademais, a solução proposta por Ozisik (1993) produz pontos críticos - oscilações inexistentes no respectivo dado experimental.

Descrição sobre os métodos aplicados sobre as operações matemáticas empregadas podem ser obtidas em Zabadal e outros (2012).

\section{REFERÊNCIAS}

AMERICAN Industrial Heat Transfer. Catálogo online, 2011. Disponível em: <http://www.aihti.com/catalog. html >. Acesso em: 1 dez. 2014.
BARTLETT, D. The fundamentals of heat exchangers. American ilnstitute of physics, 1996. Disponível em: <http://www.ewp.rpi.edu/hartford/ ernesto/ F2011/EP/MaterialsforStudents/Chan/Bartlett1996. pdf >. Acesso em: 20 maio 2014.

CARNAHAM, J. Applied numerical methods. New York: McGraw-Hill, 1990.

GREENSPAN, D.; CASULI, V. Numerical analysis for applied mathematics, science and engineering. Redwood City: Addison Wesley Publishing CO., 1988.

HOLMAN, J. Heat transfer. McGraw-Hill Series in Mechanical Engineering. São Paulo: McGraw-Hill, 2009.

MALISKA, C. Transferência de calor e mecânica dos fluídos computacional: fundamentos e coordenadas generalizadas. Rio de Janeiro: LTC, 1995.

MUKHERJEE, R. Effectively Design Shell-and-Tube Heat Exchangers. Chemical engineering progress. February, 1998.

ÖZISIK, N. Heat conduction. New York: John Wiley \& Sons, 1993.

POLYANIN, A.; ZAITSEV, V. Handbook of nonlinear partial differential equations. Boca Ratón: Chapman \& Hall/CRC, 2004.

RAFFERTY, K. D.; CULVER, G. Heat Exchangers. Geoheat center bulletin, March, 1998. Disponível em: <http://geoheat.oit.edu/bulletin/bull19-1/art4.pdf>. Acesso em: 27 set. 2014.

ZABADAL, J. GARCIA, R. RIBEIRO, V. Equações diferenciais para engenheiros: uma abordagem prática. Porto Alegre: UniRitter, 2012. 
1. Professor e Pesquisador do Centro Universitário Ritter dos Reis, Faculdade de Informática; Professor da Escola Superior de Propaganda e Marketing, grupo de Métodos Quantitativos. E-mail: vinicius@uniritter.edu.br / vinicius.ribeiro@espm.br

2. Professor e Pesquisador da Universidade Federal do Rio Grande do Sul, Departamento de Engenharia Mecânica - Grupo de Estudos Nucleares. E-mail: jorge.zabadal@ufrgs.br

3. Professor e Pesquisador da Universidade Federal do Rio Grande do Sul, Aceito em: 11 de Janeiro de 2015 Departamento de Engenharia Mecânica - Grupo de Estudos Nucleares. E-mail: borges@ufrgs.br 